\title{
Exploiting transcriptome data for the development and characterization of gene-based SSR markers related to cold tolerance in oil palm (Elaeis guineensis)
}

Yong Xiao ${ }^{{ }^{*+}}$, Lixia Zhou ${ }^{1+}$, Wei Xia ${ }^{1}$, Annaliese S Mason ${ }^{3}$, Yaodong Yang ${ }^{1}$, Zilong $\mathrm{Ma}^{2}$ and Ming Peng ${ }^{2}$

\begin{abstract}
Background: The oil palm (Elaeis guineensis, $2 n=32$ ) has the highest oil yield of any crop species, as well as comprising the richest dietary source of provitamin A. For the tropical species, the best mean growth temperature is about $27^{\circ} \mathrm{C}$, with a minimal growth temperature of $15^{\circ} \mathrm{C}$. Hence, the plantation area is limited into the geographical ranges of $10^{\circ} \mathrm{N}$ to $10^{\circ} \mathrm{S}$. Enhancing cold tolerance capability will increase the total cultivation area and subsequently oil productivity of this tropical species. Developing molecular markers related to cold tolerance would be helpful for molecular breeding of cold tolerant Elaeis guineensis.

Results: In total, 5791 gene-based SSRs were identified in 51,452 expressed sequences from Elaeis guineensis transcriptome data: approximately one SSR was detected per 10 expressed sequences. Of these 5791 gene-based SSRs, 916 were derived from expressed sequences up- or down-regulated at least two-fold in response to cold stress. A total of 182 polymorphic markers were developed and characterized from 442 primer pairs flanking these cold-responsive SSR repeats. The polymorphic information content (PIC) of these polymorphic SSR markers across 24 lines of Elaeis guineensis varied from 0.08 to 0.65 (mean = $0.31 \pm 0.12$ ). Using in-silico mapping, 137 (75.3\%) of the 182 polymorphic SSR markers were located onto the 16 Elaeis guineensis chromosomes. Total coverage of $473 \mathrm{Mbp}$ was achieved, with an average physical distance of $3.4 \mathrm{Mbp}$ between adjacent markers (range $96 \mathrm{bp}-20.8 \mathrm{Mbp}$ ). Meanwhile, Comparative analysis of transcriptome under cold stress revealed that one ICE1 putative ortholog, five CBF putative orthologs, 19 NAC transcription factors and four cold-induced orhologs were up-regulated at least two fold in response to cold stress. Interestingly, 5' untranslated region of both Unigene21287 (ICE1) and CL2628.Contig1 (NAC) both contained an SSR markers.
\end{abstract}

Conclusions: In the present study, a series of SSR markers were developed based on sequences differentially expressed in response to cold stress. These EST-SSR markers would be particularly useful for gene mapping and population structure analysis in Elaeis guineensis. Meanwhile, the EST-SSR loci were inducible expressed in response to low temperature, which may have potential application in identifying trait-associated markers in oil palm in the future.

\footnotetext{
* Correspondence: xiaoyong1980@webmail.hzau.edu.cn

${ }^{\dagger}$ Equal contributors

'Hainan Key Laboratory of Tropical Oil Crops Biology/Coconut Research Institute, Chinese Academy of Tropical Agricultural Sciences, Wenchang, Hainan 571339, P.R. China

Full list of author information is available at the end of the article
} 


\section{Background}

Oil palm (Elaeis guineensis Jacq., $2 \mathrm{n}=32$ ), belonging to the genus Elaeis in the monocotyledonous family Arecaceae (Palmaceae), is an important tropical oil crop. The genus Elaeis consists of two different species, Elaeis guineensis (African oil palm) and Elaeis oleifera (American oil palm) [1]. Elaeis guineensis is currently commercially cultivated for palm oil production in the tropics, particularly in Indonesia and Malaysia. Some efforts have been made to introduce African oil palm into subtropical regions in regional trial plantation, including in the Hainan province located in the southern China. However, winter temperatures in these regions are generally lower than $20^{\circ} \mathrm{C}$ (and can even low than $10^{\circ} \mathrm{C}$ ), which resulted in slowing of flower bud differentiation and fruit development, subsequently severely affecting the oil palm fruit productivity. Hence, enhancing cold tolerance in this tropical species is a primary breeding goal for producing African oil palm genotypes suitable for these subtropical regions.

Microsatellites (simple sequence repeats, SSRs) are tandem DNA repeats of 1-6 nucleotides per unit, and are mostly found in non-coding regions of eukaryotic genomes. Due to low selection pressure in non-coding regions, non-coding SSRs are often highly polymorphic as well as co-dominant and simple to detect. Non-coding SSRs have been widely used for the analysis of genetic diversity and population structure, construction of linkage maps, and detection of quantitative trait loci [2-5]. However, SSRs located in coding and untranslated regions (transcribed SSRs) can be efficient functional markers in genic regions [6]. SSR variation in coding regions can lead directly to functional protein changes, while SSRs occurring in $5^{\prime}$ untranslated regions (5'-UTRs) can affect transcription and translation, and SSRs in 3'-UTRs can affect splicing [7]. Thus, SSRs from transcribed sequences may be directly related to phenotypic variation, and hence functional trait markers.

Molecular markers as AFLPs, RAPDs and AFLPs have been widely used for analyzing genetic diversity and population structure, identification of trait-associated markers and genotype characterization in Elaeis guineensis [8-11]. Recently, there is increasing interest in the use of transcriptome sequencing to understand the molecular mechanisms which govern important agronomic traits in Elaeis guineensis [12]. Thus, a large number of expressed sequence tags (ESTs) were released. Obviously, this sequence information comprises a valuable resource for identifying gene-associated SSR markers in Elaeis guineensis. Previously, EST-SSRs in Elaeis guineensis based on this released data have been provided by three studies. Of these three studies, Low et al. [13] reported identification of 648 EST-SSRs associated with tissue culture, while two other studies reported EST-SSRs which were not associated with particular agronomic traits $[14,15]$.
Here, we reported our work on development and characterization of EST-SSR derived from expressed sequences up- or down-regulated at least two-fold in response to cold stress. Our study comprises five parts: (1) Characterization of the frequency and distribution of putative SSRs obtained from Elaeis guineensis transcriptome data, (2) analysis of polymorphism in the EST-SSR markers derived from expressed sequences up- or down-regulated at least two-fold in response to cold stress, (3) in-silico mapping of these polymorphic markers, (4) assessment of physical distance between these polymorphic markers and candidate genes associated with cold stress, and (5) exploring the population structure of the 192 oil palm lines using the SSR markers linked to candidate genes associated with cold stress. These SSR markers developed in the study will be useful for establishment of genetic mapping as well as population genetic studies, and will provide candidate markers for genetic improvement of cold stress in Elaeis guineensis.

\section{Methods}

\section{Plant materials}

The oil palm varieties, dura (the thick-shelled African oil palm) and pisifera (the thin-shelled African oil palm), were introduced from Malaysia to China in the 1990s and subsequently mutual crossed to produce a large number of $F_{1}$ hybrids. The plantation trial showed that a few $F_{1}$ hybrids can adapt to winter low temperature of Hainan province located on Southern China. The selected $\mathrm{F} 1$ hybrid seedlings were treated as follows: $F_{1}$ hybrid seedlings were grown in nurseries. Twenty one one-year-old F1 hybrid plants germinated in the same week and grown in the same nursery were selected for subsequently cold treatment. Prior to cold treatment, the hybrid seedling were placed in a growth chamber at $26^{\circ} \mathrm{C}$ for one day. Subsequently, spear leaf samples were collected from three individual replicates (as controls) for RNA extraction. The remaining six groups of three seedling replicates were kept at $8^{\circ} \mathrm{C}$ for 0.5 hours, 1 hour, 4 hours, 8 hours, 1 day and 7 days respectively before sampling. Spear leaves were sampled from control and cold-treated seedlings and immediately frozen in liquid nitrogen. Total RNA was extracted from the control and cold treatment samples based on the MRIP method described by Xiao et al. [16]. mRNA mixtures from the control sample and the cold-treatment sample were prepared in equal proportions for Illumina sequencing.

Moreover, 192 oil palm lines were collected from Hainan province located in Southern China (44) and from Malaysia (148). Among these oil palm individuals collected from Malaysia, 34 were produced by selfpollination of the selected $F_{1}$ plants, showing adaptation to the low winter temperatures in the Hainan province. 
The other 114 oil palm individuals were recently introduced into China, of which 29 were also produced from the self-pollination of $\mathrm{F}_{1}$ plants between dura and pisifera and for which the pedigrees of the remaining lines were unknown. DNA samples were prepared from young leaves of the 192 oil palm trees using the mini-CTAB methold [17].

\section{Illumina sequencing and de novo assembly}

Purified mRNA isolated from the control sample and from the cold-treatment mixture were separately fragmented with divalent cations under increased temperature. These short fragments were taken as templates to synthesize the first-strand cDNA using hexamer primers and superscript ${ }^{\mathrm{TM}}$ III (Invitrogen ${ }^{\mathrm{TM}}$, Carlsbad, CA, USA). Second-strand cDNA was then synthesized in a solution containing buffer, dNTP, RNaseH and DNA polymerase I and subsequently purified using a QiaQuick PCR extraction kit (Qiagen). EB buffer was used to resolve these short fragments for end reparation and poly (A) addition. The sequence adaptors were linked to two ends of short cDNA sequences and suitably sized cDNA fragments were selected out for PCR amplification based on the agrose gel electrophoresis results. Finally, the library established was sequenced using an Illumina Hiseq $^{\mathrm{Tm}}$ 2000. The paired-end library was developed according to the paired-End sample Preparation Kit protocol (Illumina, USA). The transcriptome short reads were de novo assembled software following the protocol documented by Grabherr et al. [18].

\section{Functional annotation of transcriptome data}

The transcript sequences were aligned with the NR database at a E-value threshold of $10^{-5}$ (E-value $\left.<0.00001\right)$. Subsequently, the transcript sequences were aligned by BLASTX to protein database, including Swiss-Prot, KEGG and COG. If alignment results of different databases conflicted, BLAST results from NR rather than Swiss-prot were given precedence. The WEGO software was applied to perform GO functional classification of the transcriptome [19]. The result of the GO annotation were also used for KEGG and COG analysis.

\section{Calculation of gene differential expression}

RPKM (Reads per $\mathrm{kb}$ per Million reads) was used to calculate gene expression level. The statistical significance of the differential expression was determined according to the method documented by Audic and Claverie [20]. When thousands of hypothesis tests are performed, the $p$-value suitable for a single test is not sufficient to guarantee a low rate of false discovery. Thus, an FDR (False Discovery Rate) control method was applied using multiple hypothesis testing to correct the $p$-value results [21]. Subsequently, the RPKM ratio was used to compute the fold change of gene expression for each pair of samples simultaneously. The differentially expressed genes were selected using a threshold of FDR $\leq 0.001$ and an absolute value of $\log _{2}$ ratio $\geq 1$ [22] .

\section{Identification of putative SSRs and primer design}

The software Msatfinder was used to identify putative SSRs based on the cut-off criteria of $12,8,5,5,5$ and 5 repeats for mono-, di-, tri-, tetra-, penta- and hexaucleotide motifs, respectively (http://www.bioinformatics. org/ftp/pub/msatfinder/). Subsequently, primers flanking SSRs were designed using Primer 3 software [23]. Using the software, a total of 3952 primer pairs were designed for these SSR sequences (information listed in Additional file 1). In order to evaluate polymorphisms in SSRs associated with response to cold stress, primers flanking SSRs in expressed sequences that were induced or repressed by low temperatures were used to amplify DNA isolated from the $24 \mathrm{~F}_{2}$ oil palm plants.

\section{PCR amplification and electrophoresis}

PCR amplification were performed in $10-\mu$ l reaction mixtures containing $100 \mathrm{ng}$ genomic DNA, $10 \times$ PCR buffer, $25 \mathrm{mMMgCl}_{2}$, $1 \mathrm{U}$ TaqDNA polymerase (TaKaRa, China), $0.5 \mu \mathrm{M}$ of each primer and $0.2 \mathrm{mM}$ dNTP mix, with the following program: denaturation for 5 minutes at $94^{\circ} \mathrm{C}, 35$ cycles of $94^{\circ} \mathrm{C}$ for 30 seconds, 30 seconds at $54.7^{\circ} \mathrm{C}$ and 30 seconds at $72^{\circ} \mathrm{C}$ for elongation, with a final extension of 7 minutes at $72^{\circ} \mathrm{C}$. PCR products were electrophoretically separated on $1 \%$ polyacrylamide denaturing gels and visualized by silver staining. Product sizes were determined by comparison to a 100 bp DNA ladder.

\section{The diversity analysis of the designed markers and chromosome location}

The polymorphic information content (PIC value) was calculated using a PIC calculator (http://www.liv.ac. $\mathrm{uk} / \mathrm{kempsj} / \mathrm{pic} . \mathrm{html}$ ) [24]. Using the BLAST algorithm, the chromosomal locations of the polymorphic markers were determined as follows: firstly, the expressed sequences, used to design primers for the polymorphic marker, were BLASTed against the oil palm contig sequences (BioprojectID: 192219: PRJNA192219 Elaeis guineensis); secondly, the chromosomal location of the matched contigs was further determined according to the released genome information of Singh et al. [25].

\section{Population structure}

Bayesian clustering was applied to analyze the population structure of 192 oil palm lines using the software STRUCTURE [26]. Ten independent calculations were performed for $K$ value ( $K$ set from 1 to 11 ). The length of burn-in time and replication number were both set to 
100,000 in each run. The maximum likelihood method was applied to assign every oil palm line to a cluster, and the cut-off probability was set to 0.6. The most probable number of true populations $(K)$ was identified by plotting $\Delta K$ values of $K$ from 1 to 10 in replicate runs for each $K$ and corresponded to the peak of the $\Delta K$ graph.

\section{Results}

Frequency and distribution of gene-based SSRs in the oil palm transcriptomes in response to cold stress

In our research (data unpublished), a total of 51,452 transcripts with an average length of 703 bp were obtained from oil palm transcriptomes in response to cold stress. These transcriptome data is available in TSA (Transcriptome Shotgun Assembly) database of NCBI website (Submission Number: GBSV00000000). Msatfinder identified 5,791 SSR loci located in 5034 transcript sequences (Additional file 1). Nearly one transcript sequence in $10(5034 / 51452)$ contained at least one SSR locus (Table 1). Among these microsatellites identified based on our cut-off criteria, tri-nucleotide motif types were the most abundant (2821, 48.71\%). Mono-nucleotide motifs comprised the next largest proportion (1741, 30.06\%), followed by di-nucleotide motifs $(1124,19.41 \%)$, with a minority of tetra-nucleotide $(73,1.26 \%)$, penta-nucleotide $(21,0.36 \%)$ and hexa-nucleotide motifs $(11,0.2 \%)$.

Of the 51,452 transcripts, 10,973 were up-regulated or down-regulated at least two-fold in response to cold stress. The 10,973 transcripts contained 916 identified SSR loci. Identical distribution with respect to microsatellite motif type was observed between all SSR loci identified in the 51,452 transcripts and the 916 SSR loci associated with response to cold stress (Figure 1). Of the SSR loci associated with response to cold stress, tri-nucleotide motif types were the most abundant (42.58\%), followed by mono-nucleotide (34.61\%) and di-nucleotide (20.52\%) motif types.

Comparative analysis was performed to ascertain the position within the transcript sequences of both the total SSRs and the cold-response SSRs (Figure 2). Total SSRs and cold-response SSRs both occurred mainly in UTR regions. Of the total SSRs, 1570 mono- repeats (accounting for $90.02 \%$ of the total mono-nucleotides), 1020 di-repeats (accounting for $90.75 \%$ of the total di-nucleotides), 2033 trirepeats (accounting for $79.26 \%$ of the total tri-nucleotides), 63 tetra-repeats (accounting for 91.3\% of the total tri-nucleotides), 21 penta-repeats (accounting for $100 \%$ of the total penta-nucleotides), and 11 hexa- repeats (accounting for $100 \%$ of the total hexa-nucleotides) occurred in un-translated regions (UTRs) of expressed transcripts. It should be noted that a largest portion of tri-nucleotide repeats $(532,20.74 \%)$ occurred in coding sequences (CDSs) of expressed transcripts. Compared to the total SSRs, the cold-response SSRs showed basically identical distribution within expressed transcripts. However, in cold-response SSRs, a comparative larger proportion of tetra-nucleotide $(2,25 \%)$ motif SSRs were located in coding sequences (CDSs) of expressed transcripts.

\section{Kyoto Encyclopedia of Genes and Genomes (KEGG) pathways of SSR-containing transcripts in response to cold stress}

Annotation of SSR-containing transcripts differentially regulated in response to cold stress showed that these transcripts were unevenly distributed between the different KEGG pathways (Figure 3). Of the 159 SSR-containing transcripts differentially regulated in response to cold stress which could be assigned at least one KEGG pathway, the largest proportion of SSR-containing transcripts $(58,36.48 \%)$ were classified into the Metabolic pathways (Pathway ID: ko01100). Plant hormone signal transduction (Pathway ID: ko04075) comprised the next largest proportion $(9,5.66 \%)$, followed by plant-pathogen interactions (8, 5.03\%; Pathway ID: ko03013), oxidative phosphorylation (6, 3.77\%; Pathway ID: ko00190), cutin, suberine and wax biosynthesis (6, 3.77\%;Pathway ID: ko00073), and ABC transporters (6, 3.77\%;Pathway: ko02010), with single transcripts related to botin metabolism, fatty acid metabolism, inositol phosphate metabolism, peroxisome, proteasome and RNA polymerase.

\section{Polymorphism in cold-response-associated SSR markers and chromosome positions in Elaeis guineensis}

A total of 442 primer pairs were successfully designed from the flanking sequences of cold-response-associated mono- to hexanucleotide SSR repeats. Primer pairs could not be designed for the remaining SSRs, mainly due to difficulties in obtaining sufficient flanking sequences from either side of the identified microsatellites. Subsequently, the 442 pairs of primer sequences flanking 132 mono-nucleotide repeats, 74 di-nucleotide repeats, 219 tri-nucleotide repeats, 7 tetra-nucleotide repeats, 7 penta-nucleotide repeats and 3 hexa-nucleotide repeats were synthesized to test the extent of polymorphism in the cold-response SSRs across the 24 oil palm lines. In 278 (62.9\%) of cases, PCR products could be amplified from genomic DNA. The remaining 164 primer pairs were excluded from further analysis due to lack of PCR products or due to weak amplification. Ninety-one primer pairs amplified monomorphic bands in all lines. In total, 182 (41.2\%) polymorphic microsatellite markers were identified (Figure 4), including 50 mono-nucleotide repeats, 22 di-nucleotide repeats, 102 tri-nucleotide repeats, 4 tetra-nucleotide repeats, 2 penta-nucleotide repeats, and 1 hexa-nucleotide repeat. The percentage of polymorphic mono-, di-, tri- and tetra-nucleotide repeats was $38 \%$, $30 \%, 47 \%$ and 57\%, respectively. From the 182 loci, 402 microsatellite alleles were identified with an average of 2.2 alleles per locus. Of the 402 alleles, 105 were from 
Table 1 Characteristics of 5791 SSRs identified based on transcriptome data of Elaeis guineensis

\begin{tabular}{|c|c|c|c|c|c|c|c|c|c|c|c|c|c|c|c|c|c|c|}
\hline \multirow[t]{2}{*}{ Motifs } & \multicolumn{15}{|c|}{ Repeat number } & \multirow[t]{2}{*}{ Total } & \multirow{2}{*}{$\begin{array}{l}\text { Average repeat } \\
\text { number }\end{array}$} & \multirow{2}{*}{$\begin{array}{l}\text { Average repeat } \\
\text { length }(b p)\end{array}$} \\
\hline & 5 & 6 & 7 & 8 & 9 & 10 & 11 & 12 & 13 & 14 & 15 & 16 & 17 & 18 & $>18$ & & & \\
\hline$a / t$ & - & - & - & - & - & - & - & 398 & 272 & 232 & 193 & 147 & 83 & 65 & 310 & 1700 & 15.19 & 15.19 \\
\hline $\mathrm{c} / \mathrm{g}$ & - & - & - & - & - & - & - & 10 & 8 & 5 & 0 & 2 & 4 & 0 & 12 & 41 & 15.85 & 15.85 \\
\hline tc/ga & - & - & - & 137 & 131 & 128 & 61 & 3 & 0 & 0 & 0 & 0 & 0 & 0 & 0 & 460 & 9.26 & 18.52 \\
\hline $\mathrm{ct} / \mathrm{ag}$ & - & - & - & 205 & 152 & 127 & 59 & 1 & 0 & 0 & 0 & 0 & 0 & 0 & 1 & 545 & 12.79 & 25.58 \\
\hline at & - & - & - & 14 & 10 & 5 & 5 & 0 & 0 & 0 & 0 & 0 & 0 & 0 & 0 & 34 & 9.03 & 18.06 \\
\hline ta & - & - & - & 7 & 3 & 8 & 1 & 0 & 0 & 0 & 0 & 0 & 0 & 0 & 0 & 19 & 9.16 & 18.32 \\
\hline ac/gt & - & - & - & 3 & 5 & 4 & 2 & 2 & 0 & 0 & 0 & 0 & 0 & 0 & 0 & 16 & 9.69 & 19.38 \\
\hline $\mathrm{ca} / \mathrm{tg}$ & - & - & - & 15 & 16 & 6 & 7 & 3 & 0 & 0 & 0 & 0 & 0 & 0 & 0 & 47 & 9.21 & 18.42 \\
\hline $\mathrm{cg}$ & - & - & - & 0 & 2 & 0 & 0 & 0 & 0 & 0 & 0 & 0 & 0 & 0 & 0 & 2 & 9 & 18 \\
\hline gc & - & - & - & 0 & 1 & 0 & 0 & 0 & 0 & 0 & 0 & 0 & 0 & 0 & 0 & 1 & 9 & 18 \\
\hline gag/ctc & 191 & 95 & 42 & 2 & 0 & 0 & 1 & 0 & 0 & 0 & 0 & 0 & 0 & 0 & 0 & 331 & 5.58 & 16.74 \\
\hline tgc/gca & 73 & 38 & 34 & 0 & 0 & 0 & 0 & 0 & 0 & 0 & 0 & 0 & 0 & 0 & 0 & 145 & 5.73 & 17.19 \\
\hline $\mathrm{cag} / \mathrm{ctg}$ & 63 & 44 & 39 & 2 & 0 & 0 & 0 & 0 & 0 & 0 & 0 & 0 & 0 & 0 & 0 & 148 & 5.86 & 17.58 \\
\hline $\mathrm{cgg} / \mathrm{ccg}$ & 127 & 66 & 34 & 7 & 0 & 0 & 0 & 0 & 0 & 0 & 0 & 0 & 0 & 0 & 0 & 234 & 9.22 & 27.66 \\
\hline $\mathrm{aag} / \mathrm{ctt}$ & 74 & 36 & 22 & 1 & 0 & 0 & 0 & 0 & 0 & 0 & 0 & 0 & 0 & 0 & 0 & 133 & 5.62 & 16.86 \\
\hline ggt/acc & 36 & 21 & 7 & 1 & 0 & 0 & 0 & 0 & 0 & 0 & 0 & 0 & 0 & 0 & 0 & 65 & 5.58 & 16.74 \\
\hline $\mathrm{ggc} / \mathrm{gcc}$ & 120 & 59 & 35 & 3 & 0 & 0 & 0 & 0 & 0 & 0 & 0 & 0 & 0 & 0 & 0 & 217 & 5.66 & 16.98 \\
\hline gat/atc & 25 & 17 & 3 & 0 & 0 & 0 & 0 & 0 & 0 & 0 & 0 & 0 & 0 & 0 & 0 & 45 & 5.51 & 16.53 \\
\hline tct/aga & 73 & 43 & 34 & 2 & 1 & 0 & 0 & 0 & 0 & 0 & 0 & 0 & 0 & 0 & 1 & 154 & 5.91 & 17.73 \\
\hline tga/tca & 58 & 18 & 9 & 2 & 0 & 0 & 0 & 0 & 0 & 0 & 0 & 0 & 0 & 0 & 0 & 87 & 5.48 & 16.44 \\
\hline $\mathrm{gac} / \mathrm{gtc}$ & 19 & 6 & 4 & 1 & 0 & 0 & 0 & 0 & 1 & 0 & 0 & 0 & 0 & 0 & 0 & 31 & 5.81 & 17.43 \\
\hline tcc/gga & 151 & 69 & 39 & 3 & 0 & 0 & 0 & 1 & 1 & 0 & 0 & 0 & 0 & 0 & 0 & 264 & 5.62 & 16.86 \\
\hline gaa/ttc & 86 & 27 & 19 & 4 & 0 & 0 & 0 & 0 & 0 & 0 & 0 & 0 & 0 & 0 & 0 & 136 & 5.57 & 16.71 \\
\hline cct/agg & 122 & 69 & 38 & 5 & 0 & 0 & 0 & 0 & 0 & 0 & 0 & 0 & 0 & 0 & 0 & 234 & 5.69 & 17.07 \\
\hline att/aat & 10 & 10 & 2 & 1 & 0 & 0 & 0 & 0 & 0 & 0 & 0 & 0 & 0 & 0 & 0 & 23 & 5.74 & 17.22 \\
\hline $\mathrm{aac} / \mathrm{gtt}$ & 7 & 4 & 0 & 2 & 0 & 0 & 0 & 0 & 0 & 0 & 0 & 0 & 0 & 0 & 0 & 13 & 5.77 & 17.31 \\
\hline $\mathrm{cgc} / \mathrm{gcg}$ & 61 & 39 & 22 & 3 & 0 & 1 & 0 & 0 & 0 & 0 & 0 & 0 & 0 & 0 & 0 & 126 & 5.77 & 17.31 \\
\hline ttg/caa & 12 & 11 & 2 & 0 & 0 & 0 & 0 & 0 & 0 & 0 & 0 & 0 & 0 & 0 & 0 & 25 & 5.6 & 16.8 \\
\hline $\mathrm{agc} / \mathrm{gct}$ & 40 & 23 & 31 & 2 & 0 & 0 & 0 & 0 & 0 & 0 & 0 & 0 & 0 & 0 & 0 & 96 & 5.95 & 17.85 \\
\hline atg/cat & 24 & 10 & 10 & 1 & 0 & 0 & 0 & 0 & 0 & 0 & 0 & 0 & 0 & 0 & 0 & 45 & 5.73 & 17.19 \\
\hline $\mathrm{tta} / \mathrm{taa}$ & 13 & 5 & 2 & 0 & 0 & 0 & 0 & 0 & 0 & 0 & 0 & 0 & 0 & 0 & 0 & 20 & 5.45 & 16.35 \\
\hline $\mathrm{cac} / \mathrm{gtg}$ & 52 & 9 & 15 & 3 & 0 & 0 & 0 & 0 & 0 & 0 & 0 & 0 & 0 & 0 & 0 & 79 & 5.61 & 16.83 \\
\hline $\operatorname{tgg} / \mathrm{cca}$ & 61 & 25 & 13 & 3 & 0 & 0 & 0 & 0 & 0 & 0 & 0 & 0 & 0 & 0 & 0 & 102 & 5.59 & 16.77 \\
\hline ata/tat & 8 & 1 & 3 & 0 & 0 & 0 & 0 & 0 & 0 & 0 & 0 & 0 & 0 & 0 & 0 & 12 & 5.58 & 16.74 \\
\hline $\mathrm{aca} / \mathrm{tgt}$ & 7 & 3 & 2 & 1 & 0 & 0 & 0 & 0 & 0 & 0 & 0 & 0 & 0 & 0 & 0 & 13 & 5.77 & 17.31 \\
\hline cga/tcg & 13 & 7 & 3 & 2 & 0 & 0 & 0 & 0 & 0 & 0 & 0 & 0 & 0 & 0 & 0 & 25 & 5.76 & 17.28 \\
\hline cgt/acg & 7 & 4 & 0 & 0 & 1 & 0 & 0 & 0 & 0 & 0 & 0 & 0 & 0 & 0 & 0 & 12 & 5.67 & 17.01 \\
\hline tag & 1 & 0 & 0 & 0 & 0 & 0 & 0 & 0 & 0 & 0 & 0 & 0 & 0 & 0 & 0 & 1 & 5 & 15 \\
\hline gta/tac & 2 & 1 & 0 & 1 & 0 & 0 & 0 & 0 & 0 & 0 & 0 & 0 & 0 & 0 & 0 & 4 & 6 & 18 \\
\hline agt & 1 & 0 & 0 & 0 & 0 & 0 & 0 & 0 & 0 & 0 & 0 & 0 & 0 & 0 & 0 & 1 & 5 & 15 \\
\hline tetra- & 60 & 12 & 0 & 0 & 0 & 1 & 0 & 0 & 0 & 0 & 0 & 0 & 0 & 0 & 0 & 73 & 5.23 & 20.92 \\
\hline
\end{tabular}


Table 1 Characteristics of 5791 SSRs identified based on transcriptome data of Elaeis guineensis (Continued)

\begin{tabular}{lllllllllllllllllll}
\hline penta & 20 & 1 & 0 & 0 & 0 & 0 & 0 & 0 & 0 & 0 & 0 & 0 & 0 & 0 & 0 & 21 & 5.05 & 25.25 \\
Hexa- & 8 & 1 & 0 & 0 & 1 & 0 & 1 & 0 & 0 & 0 & 0 & 0 & 0 & 0 & 0 & 11 & 6 & 36 \\
Total & 1625 & 774 & 464 & 433 & 323 & 280 & 137 & 418 & 282 & 237 & 193 & 149 & 87 & 65 & 324 & 5791 & 9.21 & 16.86 \\
\hline
\end{tabular}

mononucleotide motif loci with an average of 2 alleles per locus; 46 were from dinucleotide motif loci with an average of 2 alleles per locus, and 227 were from trinucleotide motif loci with an average of 2.2 alleles per locus. Across the 182 polymorphic markers, PIC values ranged from 0.08 to 0.65 (mean $=0.31 \pm 0.12$ ), suggesting the cold-response-associated SSR markers developed had moderate levels of polymorphism (Figure 5). The mean PICs of the 50 mono-nucleotide, 22 di-nucleotide and 102 tri-nucleotide repeats were $0.30,0.31$ and 0.31 , respectively. Detailed information for the 182 polymorphic markers is listed in Additional file 1.

Based on in-silico mapping, 137 (75.3\%) of the 182 developed gene-based SSR markers could be placed on Elaeis guineensis chromosomes (Figure 6). The number of SSR markers per chromosome varied from 3 (chromosome 9) to 20 (chromosome 5), with an average of 8.52 SSR markers per chromosome across the 16 chromosomes. The physical distance between adjacent SSR markers ranged from 96 bp to $20.8 \mathrm{Mbp}$, with a total coverage length of $473.4 \mathrm{Mbp}$ and an average physical length of $3.5 \mathrm{Mbp}$. Detailed information for the physical distance between adjacent markers had been listed in Additional file 2.
Identificaiton of candidate genes in response to cold stress and physical distance between these candidate genes and the SSR markers

The comparative analysis of transcriptomes under cold stress revealed that 10,973 transcripts were up-regulated or down-regulated at least two-fold in response to cold stress. Among these transcripts in response to cold stress, some were functional annotated as cold-resistance genes documented in the previous researches. Based on annotation results, eight $C B F$ orthologs, two ICE1 orthologs, three SIZ1 orthologs, two ZAT10 orthologs, one HOS1 orthlogs and one MYB15 orthologs were detected, comprising some crucial transcription factors involved in the CBF-mediated cold signal transduction. Of these, six transcripts (35.3\%) were up-regulated at least two fold, including Unigene21287 (ICE1, 4.49 fold), CL4558.Contig1 (CBF, 6.14 fold), CL4552.Contig2 ( $C B F$, 11.08 fold), CL83.Contig2 (CBF, 5.44 fold), CL83.Contig3 (CBF, 7.1 fold) and Unigene 26961 (CBF, 11.9 fold). Interestingly, $5^{\prime}$ untranslated region of candidate Unigene21287 (ICE1, 4.49 fold) contained a SSR loci (Unigene21287 SSR) with comparatively high diversity extent (PIC value: 0.619) across the 24 lines of Elaeis guineensis. Meanwhile, based on in-silico mapping,

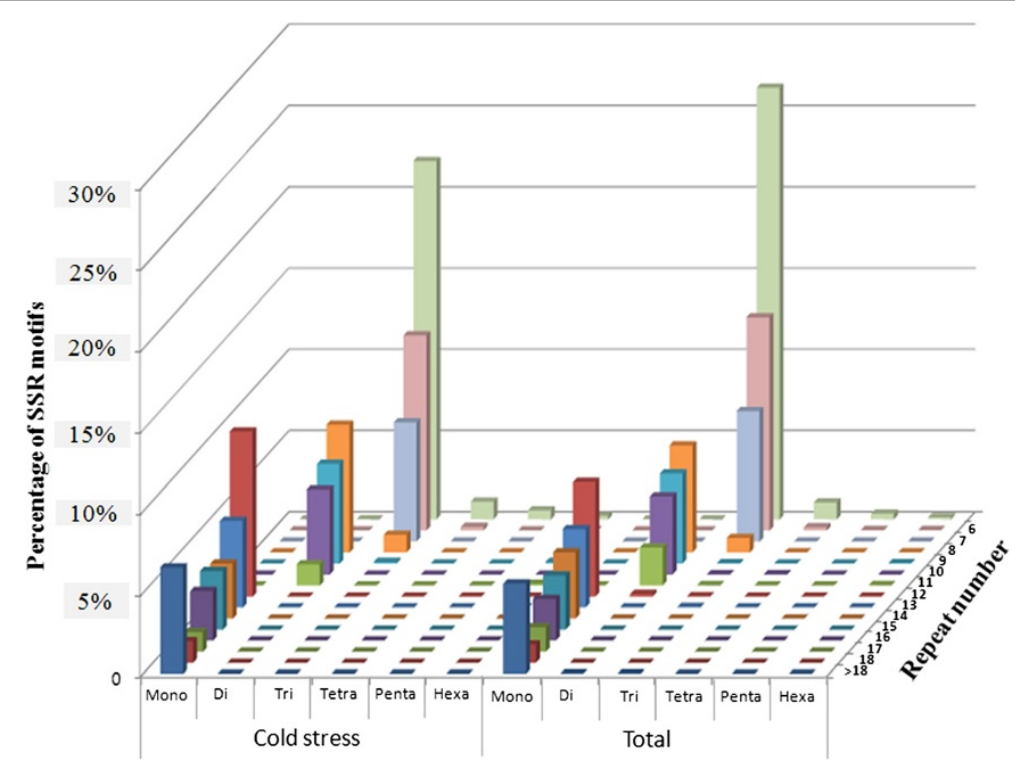

Figure 1 The distribution of the motif repeats of mono to hexa-nucleotide microsatellites based on all transcript sequences and transcript sequences differentially expressed in response to cold treatment. 


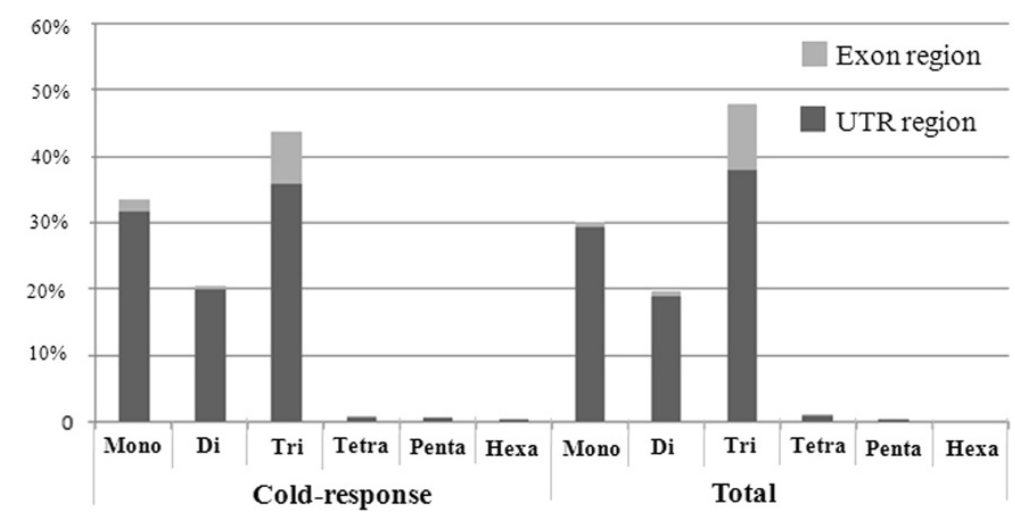

Figure 2 The percentage distribution of mono-, di-, tri-, tetra-, penta- and hexa-nucleotide repeat SSRs between UTRs and exon regions for total and cold-response-associated SSRs in African oil palm.

three of the other five candidate genes involved in CBF-mediated pathway were located on genome scaffolds containing SSR markers. The physical distance between the three candidates and adjacent SSR markers were listed in Additional file 3.

In addition, some transcripts were classified as $N A C$ transcription factors according to COG annotation results, of which some members have been documented to be related to cold tolerance in some species. In Elaeis guineensis, 19 (41.3\%) of 46 NAC transcription factors were up-regulated at least two fold under cold stress, with fold changes varying from 2.16 fold (Unigene7160) to 10.32 fold (Unigene22381). Of them, the $5^{\prime}$ untranslated region of CL2628.Contig1 (NAC, up-regulated 2.82 fold) also contained one SSR maker with moderate polymorphism
(PIC value: 0.275 ) across the 24 lines of Elaeis guineensis. Fourteen of other 18 candidate NAC transcription factors were also located on genome scaffolds containing SSR markers. The physical distances between the 15 candidates and the adjacent SSR markers are listed in Additional file 4.

Meanwhile, 36 transcripts were functionally classified as putative cold-induced putative orthologs based on annotation results due to previous documentation of cold-inducible expression in other species. However, in Elaeis guineensis, only four (10.8\%) of 37 transcripts were up-regulated at least two fold in response to low temperature, including CL3095.Contig2 (cold induced protein, 3.67 fold), CL384.Contig1 (cold induced protein, 2.89 fold), CL2052.Contig2 (cold induced protein, 3.75

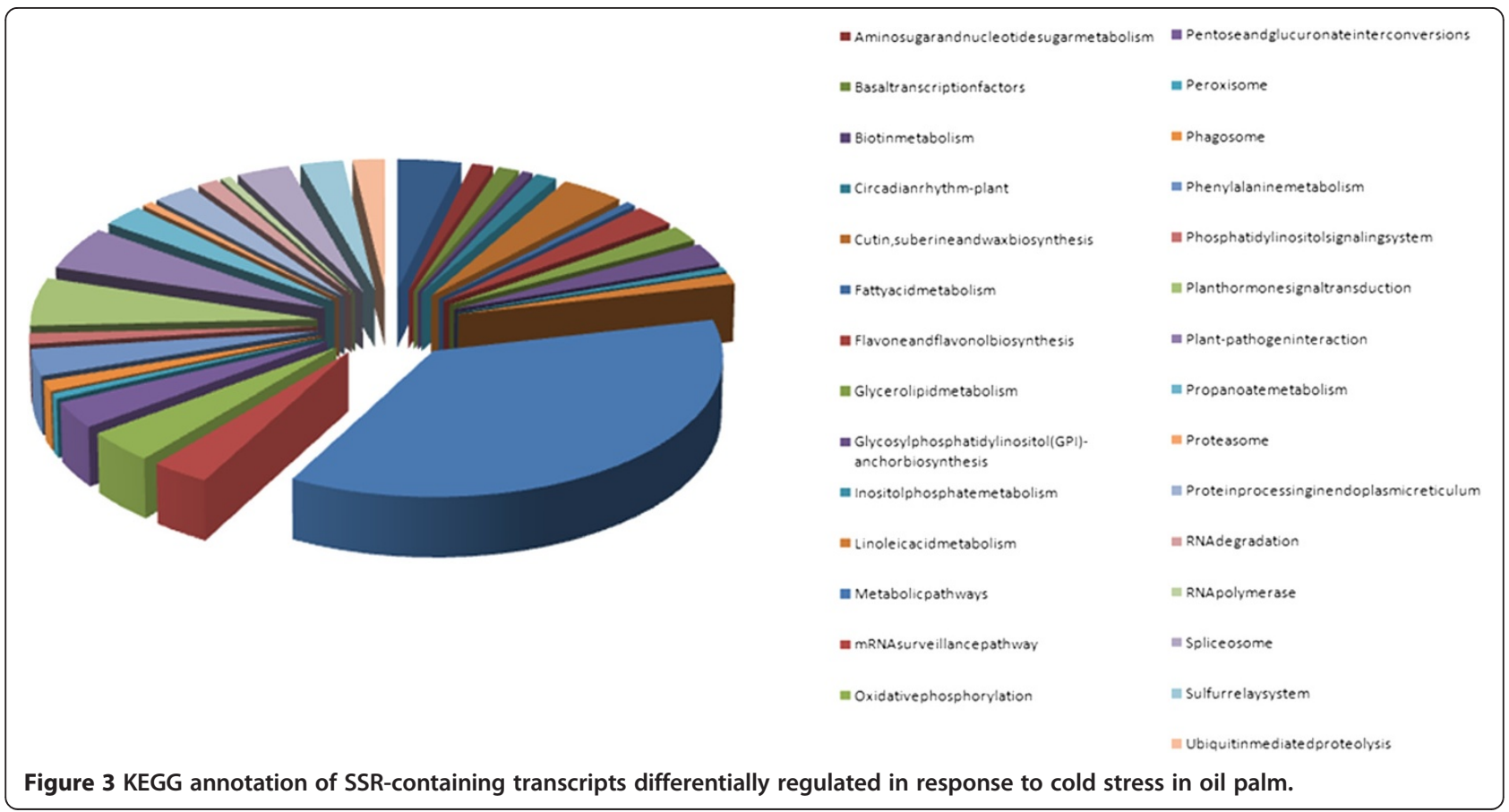



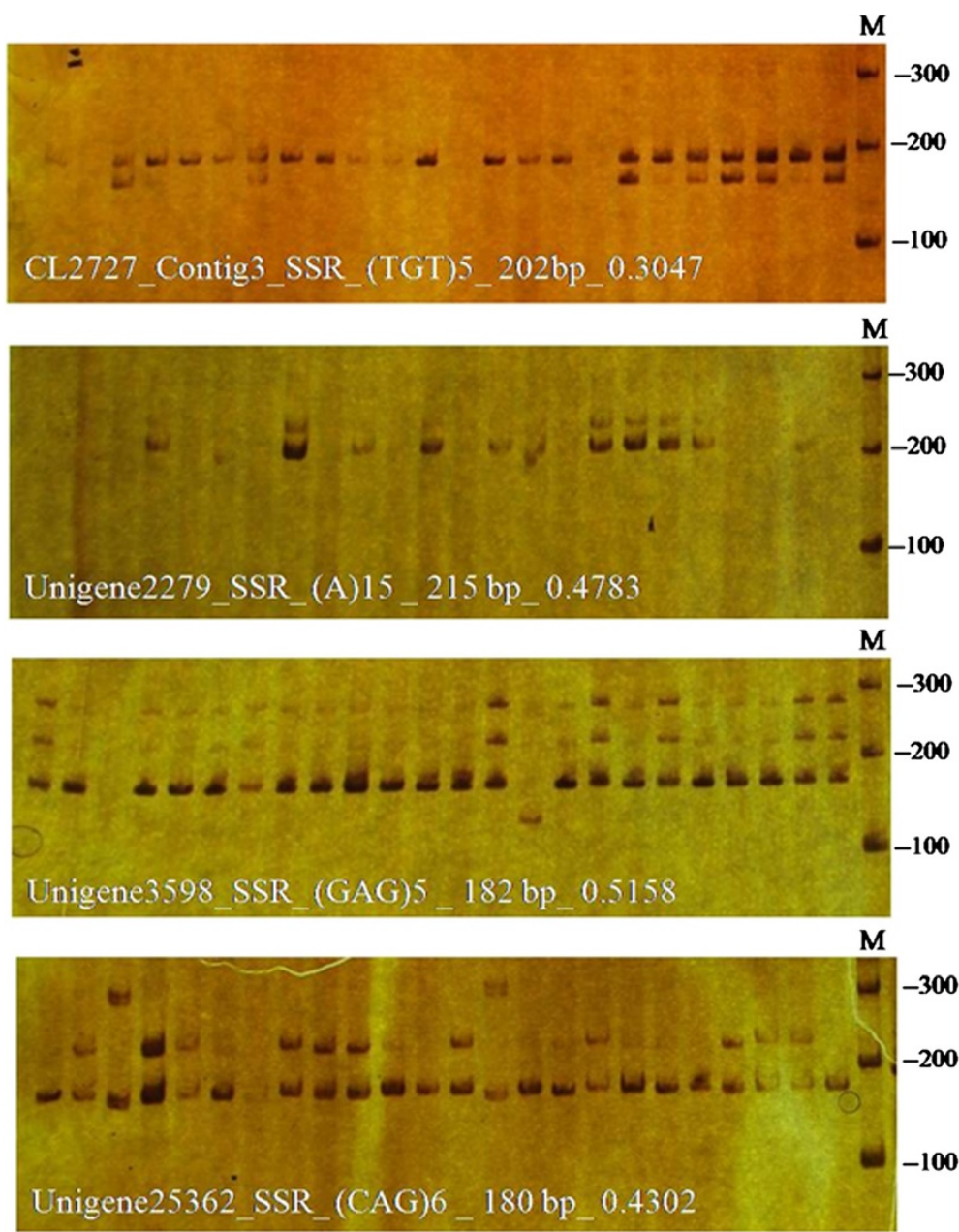

Figure 4 PCR products and polymorphic characteristics of four SSR markers across 24 Elaeis guineensis accessions.

fold) and CL559.Contig2 (cold induced protein, 2.54). of the four candidates, three were located on genome scaffolds containing SSR markers. The physical distances between the three candidates and their adjacent SSR markers are listed in Additional file 5.
Exploring population structure of 192 oil palm lines using ten SSR markers linked to candidate genes

Ten SSR markers (three closely linked with candidate genes and seven less closely linked to candidate genes, including Unigene21287_SSR, Unigene25696_SSR,

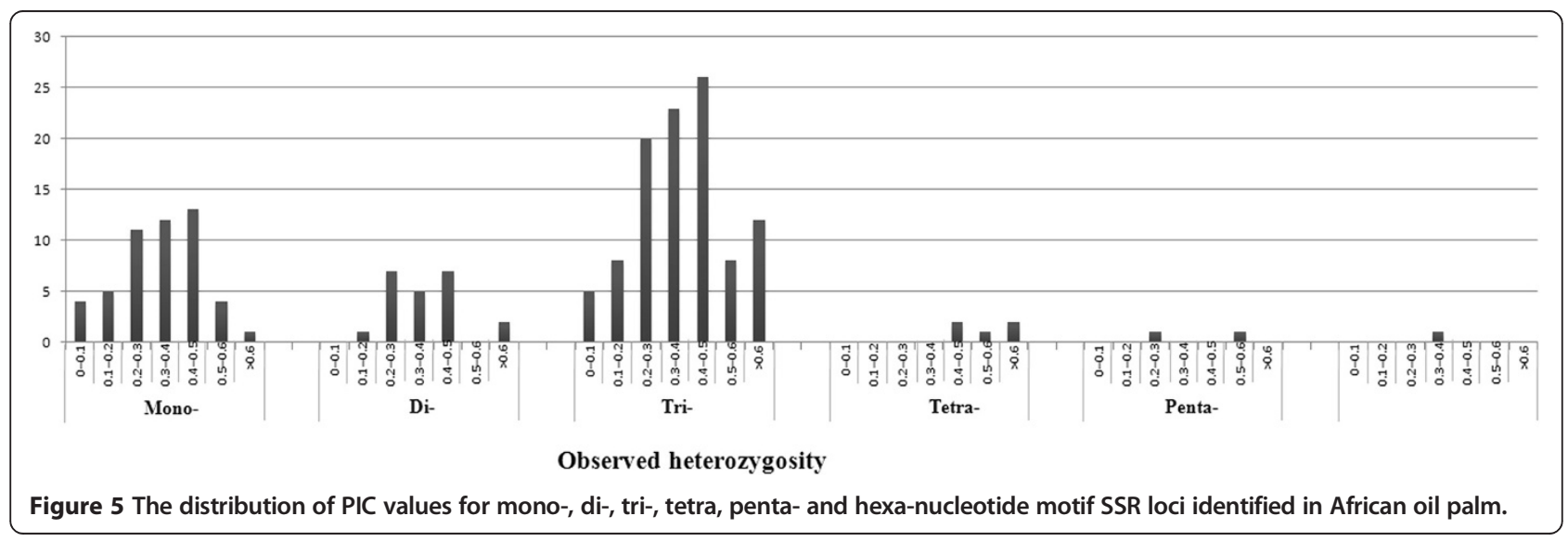




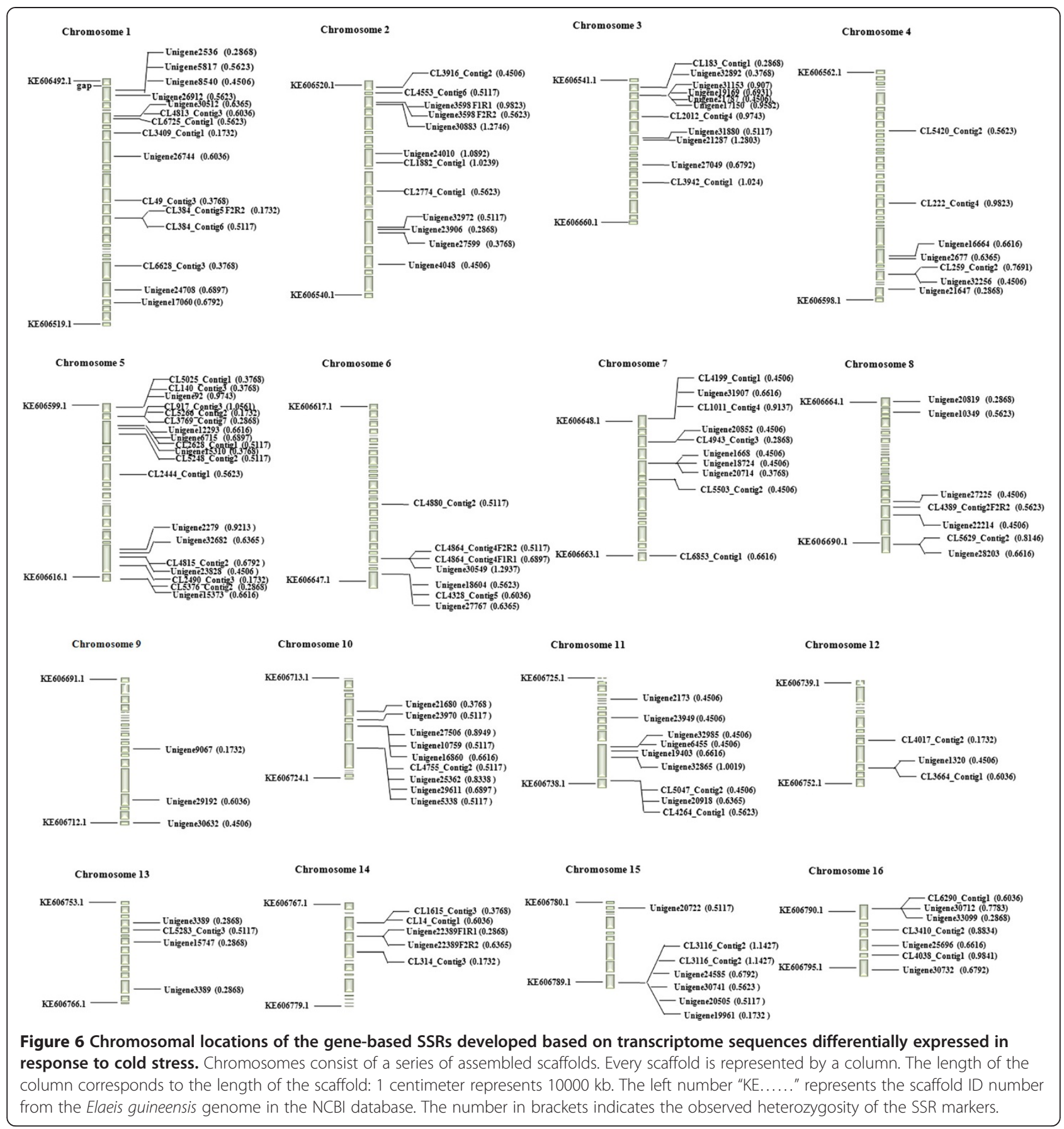

CL2628_Contig1 SSR, Unigene19403 SSR, Unigene30741 SSR, CL14_Contig1_SSR, Unigene3598_SSR, CL2490_Contig3_SSR, Unigene32985_SSR, and CL4880_Contig2_SSR) were used to genotype 192 individuals of oil palm collected from Malaysia and China. Of these, 34 lines of oil palm were selected from the $F_{2}$ population derived from self-pollination of the selected $F_{1}$ hybrid that showed adaptation to the low winter temperatures in the Hainan province and 44 were collected from the Hainan province located in Southern China. Other oil palm individuals were recently collected from Malaysia, which did not undergo selection for cold tolerance. The method of Evanno et al. [27] was applied to identify the most likely number of 'true populations' in the 192 lines of oil palm, two genetic groups were inferred (Figure 7). Structure analysis showed that there is partial separation between these oil palm lines with some cold adaptation and those without. Almost all $\mathrm{F}_{2}$ individuals resulting from self-pollination of the selected $F_{1}$ plant were exclusively clustered into the red subgroup (Figure 7). However, oil 


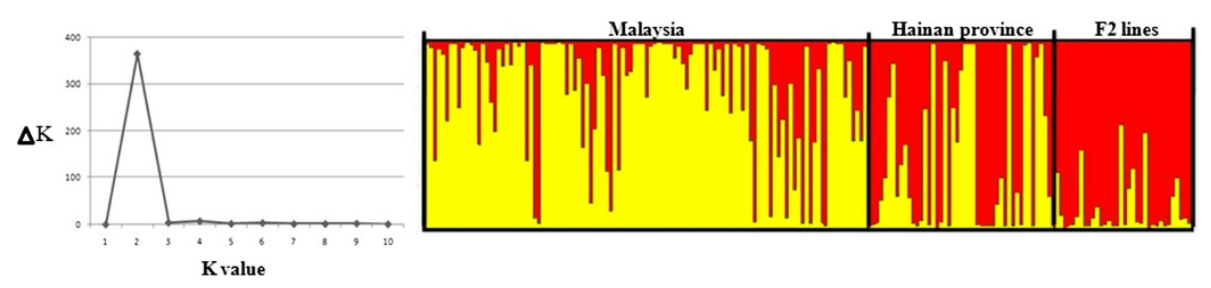

Figure 7 Population structure of 192 oil palm lines collected from the Hainan province located in Southern China and from Malaysia.

palm lines collected from Hainan province were found in both subpopulaitons: approximately half (26) were grouped into the red subpopulation. These oil palm lines may be also derived from Southeast Asia and introduced into china in the early twentieth century. Due to lack of adaptation to the climate environment in Hainan province, almost all of the oil palm lines introduced showed low productivity. Subsequently, most of these oil palm lines were cut down and the remaining oil palm lines were used only as aforestation trees. Therefore, there was not extensive artificial selection on the oil palm lines collected from Hainan province, and hence only half can been clustered together with the $F_{2}$ individuals. The majority of the oil palm lines collected from Malaysia were grouped into the yellow subgroup (Figure 7). The oil palm lines were recently introduced into China from Malaysia, and did not undergo the selection for adaptation to the Hainan climatic environment. Therefore, these oil palm lines could grouped into another subpopulation relative to the F2 individuals. In brief, these markers linked to candidate genes can partial distinguish between oil palm adapted or non-adapted to winter low temperatures in the Hainan province, suggesting that these markers may be related to cold stress.

\section{Discussions}

Elaeis guineensis has the highest oil yield of any crop species, as well as comprising the richest dietary source of provitamin A [28]. Currently, this crop can only be cultivated in tropical countries. Some effort has been made to introduce Elaeis guineensis into subtropical regions worldwide, for example the Yunnan and Hainan provinces in China. However, low winter temperature in these subtropical regions has a serious effect on the flesh fruit productivity of Elaeis guineensis. In order to facilitate improvement of cold tolerance in this important crop species, we aimed to develop molecular markers associated with cold tolerance in Elaeis guineensis. In this study, we developed 182 polymorphic EST-SSR markers based on sequences differentially expressed in response to cold stress. PIC values of these EST-SSR markers ranged from 0.08 to 0.65 (mean $=0.31 \pm 0.12$ ). Meanwhile, based on in-silico mapping, the EST-SSR markers were located on each of the 16 Elaeis guineensis chromosomes.
Subsequently, the physical distances between the developed EST-SSR markers and putative genes related to cold stress were also calculated. Therefore, the EST-SSR markers developed based on sequences differentially expressed in response to cold stress have potential application for association analysis for molecular breeding of cold tolerance in Elaeis guineensis.

In previous studies, EST-SSRs were generally identified based on sequencing of Elaeis guineensis cDNA libraries. Compared to Illumina sequencing, sequencing of cDNA libraries produces very limited expressed sequence data. Tranbarger et al. [14] identified 465 EST-SSRs from 6,103 non-redundant ESTs derived from cDNA libraries of developing vegetative and reproductive tissues in Elaeis guineensis. Of these, only 289 primer pairs flanking the EST-SSRs could be designed. Low et al. [13] identified 648 non-redundant EST-SSRs from 9584 expressed sequence tags in a total of 12 standard cDNA libraries, representing three main developmental stages in oil palm tissue culture. Ting et al. [15] identified 722 SSRs from 10258 unique sequences. In this study, we identified a total of 5,791 SSRs, a considerably greater number than identified in previous studies. Meanwhile, 3952 primer pairs were designed for these SSR sequences, which is far more than the number of SSR pairs developed in the previous studies in Elaeis guineensis [13-15]. Of these primer pairs, we focused on 442 primer pairs corresponding to the expressed sequences which were induced or repressed at least two-fold under cold stress.

Based on cut-off criteria of $12,8,5,5$, and 5 repeats for mono-, di-, tri-, tetra-, penta- and hexa-nucleotide SSRs, tri-nucleotides were the most abundant EST-SSR markers. This result is identical to previous findings of tri-nucleotide motifs as the most frequent EST-SSR motif in Cocos nucifera [29]. However, the most abundant motifs are dinucleotides in some other species [30], which may be a result of loose cut-off criteria to identify SSRs. In order to compare the overall density of SSRs in the Elaeis guineensis transcriptome with that reported in other plant species, we re-computed SSRs using the same cut-off criteria as Cardle et al. [31], with 7, 5, 4 and 4 repeats for di-, tri-, tetra- and penta-, respectively. A total of 4794 SSRs were identified with one SSR per $7.53 \mathrm{~kb}$. The SSR density in Elaeis guineensis is similar to that in coconut 
palm (one SSR per $7.59 \mathrm{~kb}$ ) and soybean (one SSR per $7.4 \mathrm{~kb})[29,32]$, but higher than in maize (one per $8.1 \mathrm{~kb}$ ), tomato (one per $11.1 \mathrm{~kb}$ ), Arabidopsis (one per $13.83 \mathrm{~kb}$ ), poplar (one per $14 \mathrm{~kb}$ ) and cotton (one per $20 \mathrm{~kb}$ ). Moreover, higher SSR density than oil palm was found in castor bean (one SSR per $1.77 \mathrm{~kb}$ ) [33,34].

Some studies have showed that SSRs are mainly located in the UTR regions of expressed sequences, especially in the case of mono-, di-, tetra-, penta-, and hexa-nucleotide motif SSRs [35]. Obviously, if the SSR (for mono-, di-, tetra-, penta-, and hexa-nucleotide motifs) is located in the coding region, mutation in the SSR sequence will cause variation in the coding frame and lead to detrimental mutations. However, a high proportion of tri-nucleotide motifs were found within the coding regions of coconut and castor bean, which may be due to the fact that copy number mutations in tri-nucleotide motifs cannot lead to frame shift mutation. We also observed a high proportion of mono- (97.09\%), di- (97.05\%), tetra- (91.3\%), penta- (100\%) and hexa-nucleotide (100\%) SSRs in UTR regions in this study. However, for tri-nucleotide motif SSRs, only 20.7\% were located in coding regions, much less than in coconut palm (53.6\%) [29] and castor bean (76.1\%) [35]. The low frequency of SSRs occurring in coding regions may indicate that coding regions are less variable and prone to mutation in Elaeis guineensis.

Identification of putative SSRs based on available expressed sequences from the NCBI databases has previously been carried out. However, the extent of polymorphism in these putative SSRs was not described in previous research [13-15]. In our study, 3952 primer pairs flanking the corresponding expressed sequences were designed based on cold-responsive transcripts of Elaeis guineensis. Of the 3952 primer pairs, 442 primer pairs flanking the expressed sequences differentially regulated in response to cold stress were used to genotype 24 lines of Elaeis guineensis. A total of 182 SSR loci were polymorphic and their PIC value ranged from 0.08 to 0.65 , with an average of 0.31 . The cold-responsive SSR markers developed in our study seem to have relatively similar levels of diversity to EST-SSRs reported in other species $[35,36]$. However, the diversity of these SSR markers was lower than previously documented genomic SSRs in other palm species [37]. This can be explained by the fact that SSRs obtained from expressed sequences undergo selection pressure against mutation due to their presence in functional genes.

Although a large number of studies have reported the development of EST-SSR markers in various plant species, chromosomal locations of these developed SSR markers is generally lacking, which is disadvantageous for subsequent studies of linkage disequilibrium, association analysis and molecular breeding. In our study, 137 (75.3\%) of the 182 markers developed were located onto the 16 chromosomes of Elaeis guineensis based on in-silico mapping, which will provide basic information for subsequent genetics and breeding studies. Moreover, plant response to low temperature is a very complex biological process, which requires integration of a large number of genes functioning together to defend against cold stress. The CBF cascade has been documented to have an important role in cold acclimation in diverse plant species. The CBF cascade involves a series of transcription factors, including ICE1, HOS1, MYB15, SIZ1 and ZAT10, transmitting cold signals and subsequently initiating immediate responses to cold stress [38]. In the present study, eight $C B F$ orthologs, two ICE1 orthologs, three SIZ1 orthologs, two ZAT10 orthologs, one HOS1 orthlogs and one MYB15 orthologs were also detected in Elaeis guineensis transcriptomes in response to low temperatures. Just like in other species, putative ICE1 and $C B F$ orthologs were also strongly induced when Elaeis guineensis suffered cold stress. Two SSR makers were closely linked separately with an ICE1 candidate (Unigene21287, up-regulated 4.49 fold) and an $C B F$ candidate (CL83.Contig3, 7.1 fold) respectively: one SSR marker was located in $5^{\prime}$ untranslated region of the ICE1 candidate and another SSR markers was only $12.6 \mathrm{~kb}$ from the $C B F$ candidate. The two candidate SSR makers should have immediate application for molecular breeding of cold tolerance in Elaeis guineensis. Meanwhile, much evidence had also revealed that $N A C$ genes also play important roles in abiotic and biotic stress responses [39]. In the study, 46 putative $N A C$ orthologs were predicted, of which 19 were up-regulated at least two fold. Among NAC candidates induced by low temperature, one SSR markers seemed to be closely linked to two NAC candidates (CL4107.Contig2 and CL2628.Contig1): located in the $5^{\prime}$ untranslated region of the NAC candidate (CL4107.Contig2) and only have $79.8 \mathrm{~kb}$ physical distance away from CBF candidate. This SSR marker closely linked to candidate genes induced by cold stress can be further validated for subsequently association analysis in Elaeis guineensis.

In addition, ten SSR markers (three closely linked with candidate genes and seven less closely linked to candidate genes) were used to analyze the population structure of 192 oil palm lines. Interesting, these markers could partial distinguish between oil palm lines that had historically undergone adaptation to climatic environment of Hainan province and those that had not. However, the structure results did not conclusively confirm the relationship between these markers and cold stress. In future, phenotypic data for the cold tolerance of the 192 oil palm lines will be investigated. Association analysis between phenotypic variations for cold stress and the SSR markers linked to candidate genes could further validate if these markers are predictive of cold tolerance in oil palm. 


\section{Conclusions}

Gene-based SSRs can directly influence phenotype and also be in close proximity to genetic variation in coding or regulatory regions corresponding to traits of interest. In the study, a total of 5,791 SSR loci were identified based transcriptome data of Elaeis guineensis separately from a control (control growth condition) RNA sample and a mixed RNA sample with cold treatment. Of these 5791 gene-based SSRs, 916 were derived from expressed sequences up- or down-regulated at least two-fold in response to cold stress. Based on the flanking sequence of the cold-reponsive SSRs, 442 primer pairs were designed and subsequently used to genotype 24 lines of Elaeis guineensis. The PCR amplification products of 182 primer pairs showed polymorphism between the 24 lines. These polymorphic markers were subsequently used for analysis of genetic diversity and population structure, identification of trait-associated markers and genotype characterization in Elaeis guineensis. Meanwhile, 137 of these SSR markers were mapped onto the 16 different chromosomes of Elaeis guineensis using in-silico mapping, which will provide basic information for location of important agronomic traits and the analysis of linkage disequibrium in Elaeis guineensis. Moreover, differential expression analysis showed that one ICE1 putative ortholog, five $C B F$ putative orthologs, 19 NAC transcription factors and four cold-induced orhologs were up-regulated at least two fold in response to cold stress. Among these, 22 candidates could be in-silico mapped on to genome scaffold containing SSR markers, of which three SSR markers were closely linked with an ICE1 candidate, a $C B F$ candidate and two $N A C$ candidates. These three candidate SSR makers would have immediate application for molecular breeding of cold tolerance in Elaeis guineensis.

\section{Additional files}

Additional file 1: The information of 3952 SSR primers, including primer sequence, Tm value, fragment size, motif, repeat number, Tm (annealing temperature), fold change, na*(Observed allele number), PIC (Polymorphic Information content) and genebank accessions.

Additional file 2: Physical distance between adjacent markers located on the same chromosome.

Additional file 3: Physical distance between markers and candidate genes involving in CBF-mediated pathway.

Additional file 4: Physical distance between SSR markers and candidate genes of NAC family.

Additional file 5: Physical distance between SSR markers and candidate genes involving in cold inducible and cold shock.

\section{Authors' contributions}

YX did the DNA extraction and subsequently PCR amplification, participated in the design of the study, performed the statistical analysis and drafted the manuscript. $L Z$ did the major experimental work including the extraction of DNA, PCR amplification and electrophoresis experiments, participated in the statistical analysis and drafted the manuscript. WX participated in data analysis and drafted the manuscript. ASM critically revised the manuscript. YY contributed to and adivised on DNA amplification experiments with some advices and participated in the design of the study. MP contributed to and adivised on DNA amplification experiments with some advices and ZM participated in the design of the study. All authors read and approved the final manuscript.

\section{Acknowledgements}

This work was supported by the Natural Science Foundation of China (No. 31101179), The Major Technology Project of Hainan (ZDZX2013023-1), an Australia Research Council Discovery Early Career Researcher Award (DE120100668), and the Natural Science Foundation of Hainan Province (313059).

\section{Author details}

${ }^{1}$ Hainan Key Laboratory of Tropical Oil Crops Biology/Coconut Research Institute, Chinese Academy of Tropical Agricultural Sciences, Wenchang, Hainan 571339, P.R. China. ${ }^{2}$ Institute of Tropical Bioscience and

Biotechnology, Chinese Academy of Tropical Agricultural Science, Haikou, Hainan 571101, P. R. China. ${ }^{3}$ School of Agriculture and Food Sciences and Centre for Integrative Legume Research, the University of Queensland, 4072 Brisbane, Australia.

Received: 8 August 2014 Accepted: 12 December 2014

Published: 19 December 2014

\section{References}

1. Corley RHV, Tinker PB: The Oil Palm. 4th edition. Oxford: UK Blackwell Science; 2003

2. Qiu D, Morgan C, Shi J, Long Y, Liu J, Li R, Zhuang X, Wang Y, Tan X, Dietrich E, Weihmann T, Everett C, Vanstraelen S, Beckett P, Fraser F, Trick M, Barnes S, Wilmer J, Schmidt R, Li J, Li D, Meng J, Bancroft I: A comparative linkage map of oilseed rape and its use for QTL analysis of seed oil and erucic acid content. Theor App/ Genet 2006, 114:67-80.

3. Xia W, Xiao Y, Liu Z, Luo Y, Mason AS, Fan H, Yang Y, Zhao S, Peng M: Development of gene-based simple sequence repeat markers for association analysis in Cocos nucifera. Mol Breeding 2014, 34:525-535.

4. Xiao Y, Chen L, Zou J, Tian E, Xia W, Meng J: Development of a population for substantial new type Brassica napus diversified at both $\mathrm{A} / \mathrm{C}$ genomes. Theor Appl Genet 2010, 121:1141-1150.

5. Xiao $Y$, Luo $Y$, Yang $Y$, Fan $H$, Xia W, Mason AS, Zhao S, Sager R, Fei Q: Development of microsatellite markers in Cocos nucifera and their application in evaluating the level of genetic diversity of Cocos nucifera. Plant Omics 2013, 6:193-200.

6. Li Y-C, Korol AB, Fahima T, Nevo E: Microsatellites within genes: structure, function, and evolution. Mol Biol Evol 2004, 21:991-1007.

7. Lawson MJ, Zhang L: Housekeeping and tissue-specific genes differ in simple sequence repeats in the 5'-UTR region. Gene 2008, 407:54-62.

8. Billotte N, Marseillac N, Risterucci AM, Adon B, Brottier P, Baurens FC, Singh R, Herran A, Asmady H, Billot C: Microsatellite-based high density linkage map in oil palm (Elaeis guineensis Jacq.). Theor Appl Genet 2005, 110:754-765.

9. Cochard B, Adon B, Rekima S, Billotte N, de Desmier CR, Koutou A, Nouy B, Omore A, Purba AR, Glazsmann JC: Geographic and genetic structure of African oil palm diversity suggests new approaches to breeding. Tree Genet Genomes 2009, 5:493-504.

10. Moretzsohn MC, Nunes CDM, Ferreira ME, Grattapaglia D: RAPD linkage mapping of the shell thickness locus in oil palm (Eleais guineensis Jacq.) Theor Appl Genet 2000, 100:63-70.

11. Rance KA, Mayes S, Price Z, Jack PL, Corley RHV: Quantitiative trait loci for yield components in oil palm (Elaeis guineensis Jacq.). Theor Appl Genet 2001, 103:1302-1310.

12. Tranbarger TJ, Dussert $S$, Joët $T$, Argout $X$, Summo M, Champion A, Cros D, Omore A, Nouy B, Morcillo F: Regulatory mechanisms underlying oil palm fruit mesocarp maturation, ripening, and functional specialization in lipid and carotenoil metabolism. Plant Physiol 2011, 156:564-584. 
13. Low ET, Alias H, Boon SH, Shariff EM, Tan CA, Ooi LC, Cheah SC, Raha AR, Wan KL, Singh R: Oil palm (Elaeis guineensis Jacq.) tissue culture ESTs: identifying genes associated with callogenesis and embryogenesis. BMC Plant Biol 2008, 8:62

14. Tranbarger TJ, Kluabmongkol W, Sangsrakru D, Morcillo F, Tregear JW, Tragoonrung S, Billotte N: SSR markers in transcripts of genes linked to post-transcriptional and transcriptional regulatory function during vegetative and reproductive development of Elaeis guineensis. BMC Plant Biol 2012, 12:1

15. Ting NC, Zaki NM, Rosli R, Low ETL, Ithnin M, Cheah SC, Tan SG, Singh R: SSR mining in oil palm EST database: application in oil palm germplasm diversity studies. J Genet 2010, 89:135-145.

16. Xiao $Y$, Yang $Y$, Cao H, Fan H, Ma Z, Lei X, Mason AS, Xia Z, Huang X: Efficient isolation of high quality RNA from tropical palms for RNA-seq analysis. Plant Omics 2012, 5:584-589.

17. Stewart $C$, Via LE: A rapid CTAB DNA isolation technique useful for RAPD fingerprinting and other PCR applications. Biotechniques 1993, 14:748-750.

18. Grabherr MG, Haas BJ, Yassour M, Levin JZ, Thompson DA, Amit I, Adiconis X, Fan L, Raychowdhury R, Zeng Q, Chen Z, Mauceli E, Hacohen N, Gnirke A Rhind N, Palma F, Birren BW, Nusbaum C, Lindblad-Toh K, Friedman N, Regev A: Full-length transcriptome assembly from RNA-seq data without a reference genome. Nat Biotechnol 2010, 29:644-652.

19. Mortazavi A, Williams BA, McCue K, Schaeffer L, Wold B: Mapping and quantifying mammalian transcriptomes by RNA-seq. Nat Methods 2008, 5:621-628.

20. Audic S, Claverie JM: The significance of digital gene expression profiles. Genome Res 1997, 7:986-995.

21. Peiner A, Yekutieli D, Benjamini Y: Identifying differentially expressed genes using false discovery rate controlling procedures. $B$ ioinformatics 2003, 19:368-375.

22. Wu J, Zhang $Y$, Zhang $H$, Huang $H$, Folta KM, Lu J: Whole genome wide expression profiles of vitis amurensis grape responding to downy mildew by using solex sequencing technology. BMC Plant Biol 2010, 10:234.

23. Rozen S, Skaletsky H: Primer3 on the WWW for General Users and for Biologist Programmers. In Bioinformatics Method and Protocols. New York: Springer; 1999:365-386.

24. Cordeiro GM, Taylor GO, Henry RJ: Characterisation of microsatellite markers from sugarcane (Saccharum sp.), a highly polyploidy species. Plant Sci 2000, 155:161-168.

25. Singh R, Ong-Abdullah M, Low ET, Manaf MA, Rosli R, Nookiah R, Ooi LC, Ooi SE, Chan KL, Halim MA, Azizi N, Nagappan J, Bacher B, Lakey N, Smith SW, He D, Hogan M, Budiman MA, Lee EK, DeSalle R, Kudrna D, Goicoechea JL, Wing RA, Wilson RK, Fulton RS, Ordway JM, Martienssen RA Sambanthamurthi R: Oil palm genome sequence reveals divergence of interfertile species in Old and New worlds. Nature 2013, 500:335-339.

26. Pritchard JK, Stephens M, Donnelly P: Inference of population structure using multilocus genotype data. Genetics 2000, 155:945-959.

27. Evanno G, Regnaut S, Goudet J: Detecting the number of clusters of individuals using the software STRUCTURE: a simulation study. Mol Ecol 2005, 14:2611-2620.

28. Murphy DJ: Oil palm: future prospects for yield and quality improvements. Lipid Technol 2009, 21:257-260.

29. Xia W, Xiao Y, Liu Z, Luo Y, Mason AS, Fan H, Yang Y, Zhao S, Peng M: Development of gene-based simple sequence repeat markers for association analysis in Cocos nucifera. Mol Breeding 2014, 34:525-535.

30. Du Q, Gong C, Pan W, Zhang D: Development and Application of Microsatellites in candidate genes related to wood properties in the Chinese white poplar (Populus tomentosa Carr.). DNA Res 2012, 20:31-44.

31. Cardle L, Ramsay L, Milbourne D, Macaulay M, Marshall D, Waugh R: Computational and experimental characterization of physically clustered simple sequence repeats in plants. Genetics 2000, 156:847-854.

32. Ellis JR, Burke JM: EST-SSRs as a resource for population genetic analysis. Heredity 2007, 99:125-132.

33. Varshney RK, Thiel T, Stein N, Langridge P, Graner A: In silico analysis on frequency and distribution of microsatellites in ESTs of some cereal species. Cell Mol Biol Lett 2002, 7:537-546.

34. Varshney RK, Graner A, Sorrells ME: Genic microsatellite markers in plants: features and applications. Trends Biotechnol 2005, 23:48-55.
35. Qiu L, Yang C, Tian B, Yang J-B, Liu A: Exploiting EST databases for the development and characterization of EST-SSR markers in castor bean (Ricinus communis L.). BMC Plant Biol 2010, 10:278.

36. Ramu P, Billot C, Rami JF, Senthilvel S, Upadhyaya HD, Reddy LA, Hash CT: Assessment of genetic diversity in the sorphum reference set using EST-SSR markers. Theor App/ Genet 2013, 126:2051-2064.

37. Rivera R, Edwards K, Barker JHA, Arnold GM, Ayad G, Hodgkin T, Karp A: Isolation and characterization of polymorphic microsatellites in Cocos nucifera L. Genome 1999, 42:668-675.

38. Chinnusamy V, Zhu J, Zhu JK: Cold stress regulation of gene expression in plants. Trends Plant Sci 2007, 12:444-451.

39. Wang X, Basnayake BM, Zhang H, Li G, Li W, Virk N, Mengiste T, Song F: The Arabidopsis ATAF1, a NAC transcription factor, is a negative regulator of defense responses against necrotrophic fungal and bacterial pathogens. Mol Plant Microbe Interact 2009, 22:1227-1238.

doi:10.1186/s12870-014-0384-2

Cite this article as: Xiao et al.: Exploiting transcriptome data for the development and characterization of gene-based SSR markers related to cold tolerance in oil palm (Elaeis guineensis). BMC Plant Biology 2014 14:384

\section{Submit your next manuscript to BioMed Central and take full advantage of:}

- Convenient online submission

- Thorough peer review

- No space constraints or color figure charges

- Immediate publication on acceptance

- Inclusion in PubMed, CAS, Scopus and Google Scholar

- Research which is freely available for redistribution 